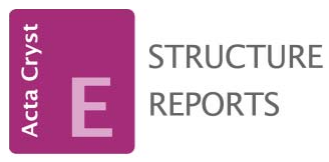

ISSN 1600-5368

Received 7 September 2014

Accepted 16 September 2014

Edited by C. Rizzoli, Universita degli Studi di Parma, Italy

Keywords: crystal structure; 1-aroylnaphthalene compound; non-coplanarly accumulated aromatic rings structure; spatial organization

CCDC reference: 1024598

Supporting information: this article has supporting information at journals.iucr.org/e

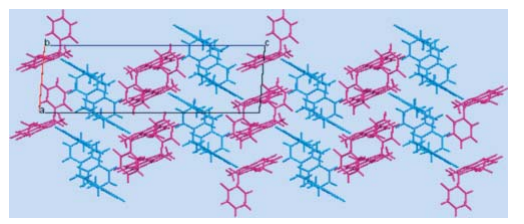

OPEN $\odot$ ACCESS

\section{Crystal structure of 1-(2-fluorobenzoyl)-2,7-di- methoxynaphthalene}

\author{
Saki Mohri, Shinji Ohisa, Takehiro Tsumuki, Noriyuki Yonezawa and Akiko \\ Okamoto*
}

Department of Organic and Polymer Materials Chemistry, Tokyo University of Agriculture \& Technology (TUAT), Koganei, Tokyo 184-8588, Japan. *Correspondence e-mail: aokamoto@cc.tuat.ac.jp

The asymmetric unit of the compound, $\mathrm{C}_{19} \mathrm{H}_{15} \mathrm{FO}_{3}$, contains two independent molecules. Each molecule has essentially the same feature of non-coplanarly accumulated aromatic rings whereby the aroyl group at the 1-position of the naphthalene ring system is twisted almost perpendicularly to the ring system [dihedral angles of $86.52(8)$ and $89.66(8)^{\circ}$ ]. In the crystal structure, molecules of the same conformer are stacked into columns parallel to the $a$ axis by van der Waals interactions only.

\section{Chemical context}

Compounds with non-coplanarly accumulated aromatic rings have received attention as unique structural building blocks from organic chemists and materials chemists, because they provide characteristic optical and electronic properties originating from their structural features. For example, biphenyl and binaphthyl are applied to optically active molecular catalysts and polymer materials on the basis of their axial chiralities (Pravas et al., 2013). In the course of our study on selective electrophilic aromatic aroylation of 2,7-dimethoxynaphthalene, it was found that peri-aroylnaphthalene compounds are formed regioselectively with the aid of suitable acidic mediators (Okamoto \& Yonezawa, 2009; Okamoto et al., 2012). The X-ray analyses of peri-aroylnaphthalene compounds revealed that the aroyl groups at the 1- and 8-positions of the naphthalene ring systems are connected almost perpendicularly but the benzene rings of the aroyl groups tilt slightly toward the exo sides of the naphthalene ring systems, as observed in 1,8-dibenzoyl-2,7-dimethoxynaphthalene (Nakaema et al., 2008) and (2,7dimethoxynaphthalene-1,8-diyl)bis(4-fluorobenzoyl)dimethanone (Watanabe et al., 2010). Moreover, the homologous 1(4-substituted benzoyl)naphthalenes also have essentially the same non-coplanar structure of the corresponding 1,8diaroylated naphthalenes, e.g. (2,7-dimethoxynaphthalen-1yl)(phenyl)methanone (Kato et al., 2010) and (2,7-dimethoxynaphthalen-1-yl)(4-fluorophenyl)methanone (Watanabe et al., 2011). On the other hand, dynamic NMR study has clarified the difference between 1-benzoylated and 1,8-dibenzoylated naphthalene (Okamoto et al., 2011). In solution, the carbon-carbon bond rotation involving the benzoyl group and the naphthalene ring system in 1,8-dibenzoyl-2,7-dimethoxynaphthalene is rather restricted, whereas the spatial organization of 1-benzoyl-2,7-dimethoxynaphthalene changes flexibly through the bond rotation. As part of our study on the molecular structures of this kind of homologous molecules, the crystal structure of title compound, a 1-benzoylated naph- 
thalene bearing the fluoro group at the 2-position of the benzoyl moiety, is discussed in this paper.<smiles>COc1ccc2ccc(OC)c(C(=O)c3ccccc3F)c2c1</smiles>

\section{Structural commentary}

There are two independent conformers in the asymmetric unit of the title compound. The independent conformers ( $A$ and $B$ ) are shown in Fig. 1. Each conformer has essentially the same non-coplanar structure. However, the orientation of the 2-fluorophenyl group against the naphthalene ring system is different in conformer $(A)$ and $(B)$, i.e., exo-side for conformer $(A)$ and endo-side for conformer $(B)$. The dihedral angle between the naphthalene ring system and the benzene ring of the 2-fluorobenzoyl group is 86.52 (8) for conformer $A$ and $89.66(8)^{\circ}$ for $B$. Bond distances and angles are not unusual.

\section{Supramolecular features}

In the crystal structure, molecules of the same conformer are stacked along the $a$ axis through weak van der Waals interactions into a columnar array (Fig. 2). No hydrogen bonds or $\pi-\pi$ stacking interactions are observed. Intra- and intercolumnar $\mathrm{C}-\mathrm{H} \cdots \pi$ contacts with an $\mathrm{H} \cdots \pi$ (centroid) separation slightly shorter than $3 \AA$ are present $(\mathrm{H} 32 \cdots \mathrm{Cg} 1=$ 2.97; H16 $\cdots C g 2^{\mathrm{i}}=2.94 ; \mathrm{H} 35 \cdots C g 3^{\mathrm{i}}=2.90 \AA ; C g 1, C g 2$ and $\mathrm{Cg} 3$ are the centroids of the $\mathrm{C} 12-\mathrm{C} 17, \mathrm{C} 1-\mathrm{C} 6$, and $\mathrm{C} 24-\mathrm{C} 29$ rings, respectively; symmetry code: (i) $1-x,-y,-z$ ), but their significance as structure-directing interactions is doubtful.

\section{Database survey}

A search of the Cambridge Structural Database (Version 5.35, last update May 2014; Allen, 2002) showed 19 and 12 structures containing the 1-substituted-2,7-dialkoxynaphthalene (including 1-acetylnaphthalene) and 1-aroyl-2,7-dialkoxynaphthalene units, respectively. The title compound has a noncoplanarly accumulated aromatic ring structure, as found in the fluoro-group-free 1-benzoylnaphthalene homologues and the fluoro-group-bearing 1-benzoylnaphthalene homologue, viz. 1-benzoyl-2,7-dimethoxynaphthalene (Kato et al., 2010) and 1-(4-fluorobenzoyl)-2,7-dimethoxynaphthalene (Watanabe et al., 2011). Both homologues form a columnar structure via $\mathrm{C}-\mathrm{H} \cdots \mathrm{O}=\mathrm{C}$ hydrogen bonds. In the case of the fluorogroup-free homologue, three conformers are found, each of them forming a columnar structure via $\mathrm{C}-\mathrm{H} \cdots \mathrm{O}=\mathrm{C}$ hydrogen bonds. The title compound forms a columnar structure similar to the homologues without $\mathrm{C}-\mathrm{H} \cdots \mathrm{O}=\mathrm{C}$ interactions in the crystal. Therefore, 1-benzoylnaphthalene homologues might be susceptible to form the columnar

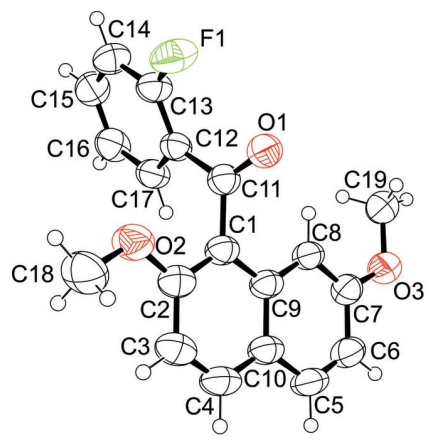

Molecule A

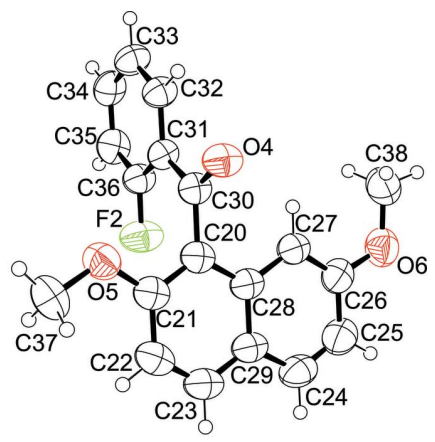

Molecule B
Figure 1

The molecular structure of the two conformers of the title compound, with displacement ellipsoids drawn at the $50 \%$ probability level.

structure. The $\mathrm{C}-\mathrm{H} \cdots \mathrm{O}=\mathrm{C}$ hydrogen bonds plausibly contribute to pack the molecules densely within the column, as indicated by the densities of the title compound $\left(1342 \mathrm{Mg} \mathrm{m}^{-3}\right)$ and the 4-fluorobenzoyl group-bearing homologue $\left(1.351 \mathrm{Mg} \mathrm{m}^{-3}\right)$. However, the number of conformers seems to afford a larger influence on the whole of the crystal packing. When several types of conformer are formed, intracolumnar interactions should be enhanced. In other words, intercolumnar interactions relatively weaken compared with the intracolumnar interactions. Consequently, the densities are apparently different between the title compound and the fluoro-group-free homologue $\left(1.276 \mathrm{Mg} \mathrm{m}^{-3}\right)$.

\section{Synthesis and crystallization}

To a test-tube-type flask, 2-fluorobenzoyl chloride $(1.1 \mathrm{mmol}$, $0.130 \mathrm{ml})$, aluminium chloride $\left(\mathrm{AlCl}_{3} ; 1.3 \mathrm{mmo} 1,0.173 \mathrm{~g}\right)$, and methylenechloride $\left(\mathrm{CH}_{2} \mathrm{Cl}_{2} ; 2.0 \mathrm{ml}\right)$ were placed and stirred at $273 \mathrm{~K}$. To the reaction mixture thus obtained 2,7-dimethoxynaphthalene $(1.0 \mathrm{mmol}, 0.188 \mathrm{~g})$ was added. After the reaction mixture had been stirred at $273 \mathrm{~K}$ for $4 \mathrm{~h}$, it was poured into methanol $(10 \mathrm{ml})$ and water $(20 \mathrm{ml})$ and the mixture was extracted with $\mathrm{CHCl}_{3}(10 \mathrm{ml} \times 3)$. The combined extracts were washed with aqueous $2 M \mathrm{NaOH}$ followed by washing with brine. The organic layers obtained were dried over

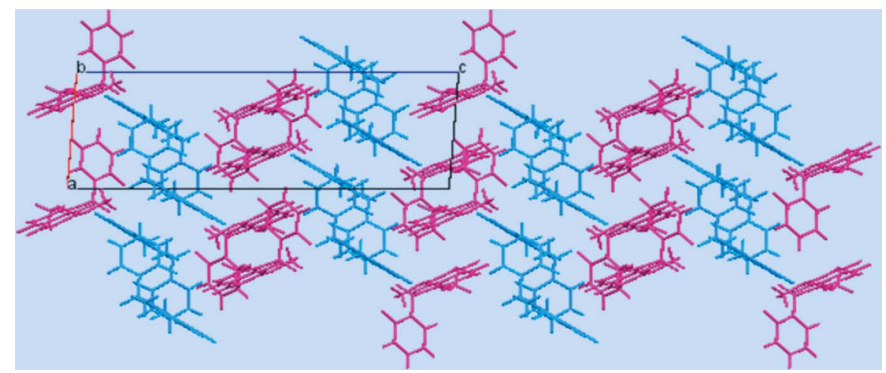

Figure 2

Crystal packing of the title compound viewed along the $b$ axis. Conformers $A$ and $B$ are drawn in purple and blue, respectively. 
Table 1

Experimental details.

\begin{tabular}{ll}
\hline Crystal data & \\
Chemical formula & $\mathrm{C}_{19} \mathrm{H}_{15} \mathrm{FO}_{3}$ \\
$M_{\mathrm{r}}$ & 310.31 \\
Crystal system, space group & Monoclinic, $P 2_{1} / n$ \\
Temperature (K) & 193 \\
$a, b, c(\AA)$ & $8.36074(15), 15.5479(3)$, \\
& $23.6898(4)$ \\
$\beta\left({ }^{\circ}\right)$ & $94.163(1)$ \\
$V\left(\AA^{3}\right)$ & $3071.36(10)$ \\
$Z$ & 8 \\
Radiation type & $\mathrm{Cu} \mathrm{K \alpha}$ \\
$\mu\left(\mathrm{mm}^{-1}\right)$ & 0.82 \\
Crystal size (mm) & $0.50 \times 0.30 \times 0.20$ \\
& \\
Data collection & Rigaku R-AXIS RAPID \\
Diffractometer & Numerical $(N U M A B S ;$ Higashi, \\
Absorption correction & $1999)$ \\
& $0.686,0.854$ \\
$T_{\text {min }}, T_{\text {max }}$ & $54661,5600,4226$ \\
No. of measured, independent and & \\
$\quad$ observed $[I>2 \sigma(I)]$ reflections & 0.032 \\
$R_{\text {int }}$ & 0.602 \\
$(\text { sin } \theta / \lambda)_{\text {max }}\left(\AA^{-1}\right)$ & \\
Refinement & \\
$R\left[F^{2}>2 \sigma\left(F^{2}\right)\right], w R\left(F^{2}\right), S$ & $0.043,0.125,1.04$ \\
No. of reflections & 5600 \\
No. of parameters & 420 \\
H-atom treatment & H-atom parameters constrained \\
$\Delta \rho_{\text {max }}, \Delta \rho_{\text {min }}\left(\mathrm{e} \AA^{-3}\right)$ & $0.29,-0.20$ \\
\hline
\end{tabular}

Computer programs: PROCESS-AUTO (Rigaku, 1998), CrystalStructure (Rigaku, 2010), SIR2004 (Burla et al., 2007), SHELXL97 (Sheldrick, 2008) and ORTEPIII (Burnett \& Johnson, 1996)

anhydrous $\mathrm{MgSO}_{4}$. The solvent was removed under reduced pressure to give a cake. The crude product was purified by recrystallization from hexane (isolated yield 63\%). Single crystals suitable for X-ray analysis were obtained from the isolated product by slow evaporation of a $\mathrm{CHCl}_{3} /$ hexane $(1: 3$ $v / v)$ solution.

${ }^{1} \mathrm{H}$ NMR $\delta\left(300 \mathrm{MHz}, \mathrm{CDCl}_{3}\right): 3.75(3 \mathrm{H}, s), 3.78(3 \mathrm{H}, s)$, $7.07(4 \mathrm{H}, m), 7.19(1 \mathrm{H}, t, J=7.6 \mathrm{~Hz}), 7.51(1 \mathrm{H}, m), 7.74(2 \mathrm{H}$, $m), 7.86(1 \mathrm{H}, d, J=8.7 \mathrm{~Hz})$ p.p.m. ${ }^{13} \mathrm{C} \mathrm{NMR} \delta(75 \mathrm{MHz}$, $\left.\mathrm{CDCl}_{3}\right): 31.19,31.23,53.95,55.30,56.47,60.94,76.71,77.13$, $77.55,102.05,110.37,116.84,117.25,124.19,124.24,124.60$, 129.83, 131.49, 131.87, 134.41, 155.94, 159.30, 159.97, 163.40, 194.56 p.p.m. IR (KBr): $1668(\mathrm{C}=\mathrm{O}), 1605,1511,1479$ (Ar, naphthalene), $1233(=\mathrm{C}-\mathrm{O}-\mathrm{C}) \mathrm{cm}^{-1}$. HRMS $(\mathrm{m} / \mathrm{z}):[M+$ $\mathrm{H}]^{+}$Calculated for $\mathrm{C}_{19} \mathrm{H}_{15} \mathrm{FO}_{3}, 310.1042$; found, 310.1005; m.p. $=365.2-365.7 \mathrm{~K}$.

\section{Refinement}

Crystal data, data collection and structure refinement details are summarized in Table 1 . All $\mathrm{H}$ atoms were located in a difference Fourier map and were subsequently refined as riding atoms, with $\mathrm{C}-\mathrm{H}=0.95-0.98 \AA$, and with $U_{\text {iso }}(\mathrm{H})=1.2$ $U_{\text {eq }}(\mathrm{C})$. The positions of methyl $\mathrm{H}$ atoms were rotationally optimized.

\section{Acknowledgements}

This work was supported by the Ogasawara Foundation for the Promotion of Science Engineering, Tokyo, Japan.

\section{References}

Allen, F. H. (2002). Acta Cryst. B58, 380-388.

Burla, M. C., Caliandro, R., Camalli, M., Carrozzini, B., Cascarano, G. L., De Caro, L., Giacovazzo, C., Polidori, G., Siliqi, D. \& Spagna, R. (2007). J. Appl. Cryst. 40, 609-613.

Burnett, M. N. \& Johnson, C. K. (1996). ORTEPIII. Report ORNL6895. Oak Ridge National Laboratory. Tennessee, USA.

Higashi, T. (1999). NUMABS. Rigaku Corporation, Tokyo, Japan.

Kato, Y., Nagasawa, A., Hijikata, D., Okamoto, A. \& Yonezawa, N. (2010). Acta Cryst. E66, o2659.

Nakaema, K., Watanabe, S., Okamoto, A., Noguchi, K. \& Yonezawa, N. (2008). Acta Cryst. E64, o807.

Okamoto, A., Mitsui, R., Oike, H. \& Yonezawa, N. (2011). Chem. Lett. 40, 1283-1284.

Okamoto, A., Watanabe, S., Nakaema, K. \& Yonezawa, N. (2012). Cryst. Struct. Theory Appl. 1, 121-127.

Okamoto, A. \& Yonezawa, N. (2009). Chem. Lett. 38, 914-915.

Pravas, D., Christopher, D., Von, B., Jean-Hubert, O., Amar, S. K., Jeffery, G. S. \& Michael, J. T. (2013). J. Am. Chem. Soc. 135, 1622016234.

Rigaku (1998). PROCESS-AUTO. Rigaku Corporation, Tokyo, Japan.

Rigaku (2010). CrystalStructure. Rigaku Corporation, Tokyo, Japan. Sheldrick, G. M. (2008). Acta Cryst. A64, 112-122.

Watanabe, S., Muto, T., Nagasawa, A., Okamoto, A. \& Yonezawa, N. (2011). Acta Cryst. E67, o1466.

Watanabe, S., Nagasawa, A., Okamoto, A., Noguchi, K. \& Yonezawa, N. (2010). Acta Cryst. E66, o329. 


\section{supporting information}

Acta Cryst. (2014). E70, 278-280 [doi:10.1107/S1600536814020807]

\section{Crystal structure of 1-(2-fluorobenzoyl)-2,7-dimethoxynaphthalene}

\section{Saki Mohri, Shinji Ohisa, Takehiro Tsumuki, Noriyuki Yonezawa and Akiko Okamoto}

\section{Computing details}

Data collection: PROCESS-AUTO (Rigaku, 1998); cell refinement: PROCESS-AUTO (Rigaku, 1998); data reduction: CrystalStructure (Rigaku, 2010); program(s) used to solve structure: SIR2004 (Burla et al., 2007); program(s) used to refine structure: SHELXL97 (Sheldrick, 2008); molecular graphics: ORTEPIII (Burnett \& Johnson, 1996); software used to prepare material for publication: SHELXL97 (Sheldrick, 2008).

\section{1-(2-Fluorobenzoyl)-2,7-dimethoxynaphthalene}

Crystal data

$\mathrm{C}_{19} \mathrm{H}_{15} \mathrm{FO}_{3}$

$M_{r}=310.31$

Monoclinic, $P 2_{1} / n$

Hall symbol: -P 2yn

$a=8.36074$ (15) $\AA$

$b=15.5479(3) \AA$

$c=23.6898(4) \AA$

$\beta=94.163(1)^{\circ}$

$V=3071.36(10) \AA^{3}$

$Z=8$

\section{Data collection}

Rigaku R-AXIS RAPID diffractometer

Radiation source: fine-focus sealed tube Graphite monochromator

Detector resolution: 10.000 pixels $\mathrm{mm}^{-1}$ $\omega$ scans

Absorption correction: numerical (NUMABS; Higashi, 1999)

$T_{\text {min }}=0.686, T_{\max }=0.854$

Refinement

Refinement on $F^{2}$

Least-squares matrix: full

$R\left[F^{2}>2 \sigma\left(F^{2}\right)\right]=0.043$

$w R\left(F^{2}\right)=0.125$

$S=1.04$

5600 reflections

420 parameters

0 restraints

Primary atom site location: structure-invariant direct methods
$F(000)=1296$

$D_{\mathrm{x}}=1.342 \mathrm{Mg} \mathrm{m}^{-3}$

$\mathrm{Cu} K \alpha$ radiation, $\lambda=1.54187 \AA$

Cell parameters from 43860 reflections

$\theta=3.4-68.2^{\circ}$

$\mu=0.82 \mathrm{~mm}^{-1}$

$T=193 \mathrm{~K}$

Block, colorless

$0.50 \times 0.30 \times 0.20 \mathrm{~mm}$

54661 measured reflections

5600 independent reflections

4226 reflections with $I>2 \sigma(I)$

$R_{\text {int }}=0.032$

$\theta_{\max }=68.2^{\circ}, \theta_{\min }=3.4^{\circ}$

$h=-9 \rightarrow 9$

$k=-18 \rightarrow 18$

$l=-28 \rightarrow 28$

Secondary atom site location: difference Fourier map

Hydrogen site location: inferred from neighbouring sites

$\mathrm{H}$-atom parameters constrained

$w=1 /\left[\sigma^{2}\left(F_{\mathrm{o}}^{2}\right)+(0.0592 P)^{2}+0.6968 P\right]$

where $P=\left(F_{\mathrm{o}}{ }^{2}+2 F_{\mathrm{c}}{ }^{2}\right) / 3$

$(\Delta / \sigma)_{\max }=0.001$

$\Delta \rho_{\max }=0.29 \mathrm{e} \AA^{-3}$

$\Delta \rho_{\min }=-0.20$ e $\AA^{-3}$ 
Extinction correction: SHELXL,

$\mathrm{Fc}^{*}=\mathrm{kFc}\left[1+0.001 \mathrm{xFc}^{2} \lambda^{3} / \sin (2 \theta)\right]^{-1 / 4}$

Extinction coefficient: $0.00104(13)$

Special details

Geometry. All e.s.d.'s (except the e.s.d. in the dihedral angle between two 1.s. planes) are estimated using the full covariance matrix. The cell e.s.d.'s are taken into account individually in the estimation of e.s.d.'s in distances, angles and torsion angles; correlations between e.s.d.'s in cell parameters are only used when they are defined by crystal symmetry. An approximate (isotropic) treatment of cell e.s.d.'s is used for estimating e.s.d.'s involving 1.s. planes.

Refinement. Refinement of $F^{2}$ against ALL reflections. The weighted $R$-factor $w R$ and goodness of fit $S$ are based on $F^{2}$, conventional $R$-factors $R$ are based on $F$, with $F$ set to zero for negative $F^{2}$. The threshold expression of $F^{2}>\sigma\left(F^{2}\right)$ is used only for calculating $R$-factors(gt) $e t c$. and is not relevant to the choice of reflections for refinement. $R$-factors based on $F^{2}$ are statistically about twice as large as those based on $F$, and $R$ - factors based on ALL data will be even larger.

Fractional atomic coordinates and isotropic or equivalent isotropic displacement parameters $\left(\AA^{2}\right)$

\begin{tabular}{|c|c|c|c|c|}
\hline & $x$ & $y$ & $z$ & $U_{\text {iso }} * / U_{\text {eq }}$ \\
\hline F1 & 0.46395 (14) & $0.11731(9)$ & $0.64805(5)$ & $0.0784(4)$ \\
\hline $\mathrm{F} 2$ & $0.21171(14)$ & $0.44622(9)$ & $0.80466(5)$ & $0.0770(4)$ \\
\hline $\mathrm{O} 1$ & $0.68735(16)$ & $0.23173(9)$ & $0.61437(5)$ & $0.0624(4)$ \\
\hline $\mathrm{O} 2$ & $0.6461(2)$ & $0.10831(9)$ & $0.49564(6)$ & $0.0736(4)$ \\
\hline $\mathrm{O} 3$ & $0.69794(18)$ & $0.56520(9)$ & $0.55258(6)$ & $0.0679(4)$ \\
\hline $\mathrm{O} 4$ & $0.47768(15)$ & $0.39636(9)$ & $0.66732(5)$ & $0.0615(4)$ \\
\hline O5 & $0.44069(18)$ & $0.59221(9)$ & $0.72372(6)$ & $0.0688(4)$ \\
\hline O6 & $0.6323(2)$ & $0.16777(10)$ & $0.83123(6)$ & $0.0773(4)$ \\
\hline $\mathrm{C} 1$ & $0.6664(2)$ & $0.25474(12)$ & $0.51551(7)$ & $0.0506(4)$ \\
\hline $\mathrm{C} 2$ & $0.6927(2)$ & 0.18855 (13) & $0.47863(8)$ & $0.0581(5)$ \\
\hline $\mathrm{C} 3$ & $0.7666(3)$ & $0.20342(15)$ & $0.42802(9)$ & $0.0667(6)$ \\
\hline $\mathrm{H} 3$ & 0.7845 & 0.1573 & 0.4029 & $0.080^{*}$ \\
\hline $\mathrm{C} 4$ & $0.8120(2)$ & $0.28474(15)$ & $0.41561(9)$ & $0.0648(6)$ \\
\hline $\mathrm{H} 4$ & 0.8624 & 0.2946 & 0.3815 & $0.078^{*}$ \\
\hline $\mathrm{C} 5$ & $0.8356(2)$ & $0.43996(15)$ & $0.43901(8)$ & $0.0644(5)$ \\
\hline H5 & 0.8902 & 0.4502 & 0.4059 & $0.077^{*}$ \\
\hline C6 & $0.8059(3)$ & $0.50630(15)$ & $0.47336(9)$ & $0.0653(6)$ \\
\hline H6 & 0.8397 & 0.5626 & 0.4642 & $0.078^{*}$ \\
\hline $\mathrm{C} 7$ & $0.7247(2)$ & $0.49263(13)$ & $0.52286(8)$ & $0.0574(5)$ \\
\hline $\mathrm{C} 8$ & $0.6796(2)$ & $0.41099(12)$ & $0.53769(7)$ & $0.0517(4)$ \\
\hline $\mathrm{H} 8$ & 0.6274 & 0.4022 & 0.5715 & $0.062 *$ \\
\hline $\mathrm{C} 9$ & $0.7109(2)$ & $0.34018(12)$ & $0.50253(7)$ & 0.0507 (4) \\
\hline $\mathrm{C} 10$ & $0.7868(2)$ & $0.35504(14)$ & $0.45149(8)$ & $0.0559(5)$ \\
\hline C11 & $0.5998(2)$ & $0.23311(12)$ & $0.57105(7)$ & $0.0496(4)$ \\
\hline $\mathrm{C} 12$ & $0.4255(2)$ & $0.21331(12)$ & $0.57099(7)$ & $0.0476(4)$ \\
\hline $\mathrm{C} 13$ & $0.3632(2)$ & $0.15833(13)$ & $0.60998(8)$ & $0.0547(5)$ \\
\hline $\mathrm{C} 14$ & $0.2027(2)$ & $0.13971(14)$ & $0.61020(9)$ & $0.0625(5)$ \\
\hline H14 & 0.1650 & 0.1007 & 0.6371 & $0.075^{*}$ \\
\hline $\mathrm{C} 15$ & $0.0981(2)$ & $0.17842(15)$ & $0.57095(9)$ & $0.0652(5)$ \\
\hline H15 & -0.0135 & 0.1668 & 0.5709 & $0.078^{*}$ \\
\hline $\mathrm{C} 16$ & 0.1535 (2) & $0.23433(15)$ & $0.53132(8)$ & $0.0643(5)$ \\
\hline H16 & 0.0803 & 0.2612 & 0.5043 & $0.077^{*}$ \\
\hline $\mathrm{C} 17$ & 0.3159 (2) & $0.25097(13)$ & $0.53125(8)$ & $0.0558(5)$ \\
\hline
\end{tabular}




\begin{tabular}{|c|c|c|c|c|}
\hline H17 & 0.3536 & 0.2887 & 0.5036 & $0.067^{*}$ \\
\hline $\mathrm{C} 18$ & $0.6836(4)$ & $0.03622(16)$ & $0.46183(11)$ & $0.0900(8)$ \\
\hline $\mathrm{H} 18 \mathrm{~A}$ & 0.6311 & 0.0428 & 0.4237 & $0.108^{*}$ \\
\hline H18B & 0.6455 & -0.0166 & 0.4790 & $0.108^{*}$ \\
\hline $\mathrm{H} 18 \mathrm{C}$ & 0.8000 & 0.0329 & 0.4595 & $0.108^{*}$ \\
\hline C19 & $0.6063(3)$ & $0.55635(15)$ & $0.60080(9)$ & $0.0675(6)$ \\
\hline H19A & 0.6662 & 0.5214 & 0.6296 & $0.081 *$ \\
\hline H19B & 0.5041 & 0.5282 & 0.5895 & $0.081^{*}$ \\
\hline $\mathrm{H} 19 \mathrm{C}$ & 0.5859 & 0.6133 & 0.6164 & $0.081 *$ \\
\hline $\mathrm{C} 20$ & $0.5035(2)$ & 0.45589 (13) & $0.75890(8)$ & 0.0518 \\
\hline $\mathrm{C} 21$ & $0.5205(2)$ & $0.54346(13)$ & $0.76503(8)$ & $0.0553(5)$ \\
\hline $\mathrm{C} 22$ & $0.6132(2)$ & $0.57851(14)$ & $0.81180(9)$ & $0.0625(5)$ \\
\hline $\mathrm{H} 22$ & 0.6273 & 0.6390 & 0.8152 & $0.075^{*}$ \\
\hline $\mathrm{C} 23$ & 0.6827 (2) & $0.52424(15)$ & $0.85231(9)$ & $0.0637(6)$ \\
\hline $\mathrm{H} 23$ & 0.7432 & 0.5478 & 0.8841 & $0.076^{*}$ \\
\hline $\mathrm{C} 24$ & $0.7374(3)$ & $0.37551(17)$ & $0.88765(9)$ & $0.0707(6)$ \\
\hline H24 & 0.7963 & 0.3974 & 0.9203 & $0.085^{*}$ \\
\hline $\mathrm{C} 25$ & $0.7240(3)$ & $0.28997(18)$ & $0.88072(9)$ & $0.0745(6)$ \\
\hline $\mathrm{H} 25$ & 0.7740 & 0.2523 & 0.9082 & $0.089^{*}$ \\
\hline $\mathrm{C} 26$ & $0.6366(2)$ & $0.25619(14)$ & $0.83326(8)$ & $0.0606(5)$ \\
\hline C27 & $0.5636(2)$ & $0.30798(13)$ & $0.79316(8)$ & $0.0542(5)$ \\
\hline $\mathrm{H} 27$ & 0.5044 & 0.2839 & 0.7613 & $0.065^{*}$ \\
\hline $\mathrm{C} 28$ & $0.5765(2)$ & $0.39972(13)$ & $0.79942(7)$ & 0.0513 \\
\hline $\mathrm{C} 29$ & $0.6658(2)$ & 0.43405 (14) & $0.84741(8)$ & $0.0568(5)$ \\
\hline $\mathrm{C} 30$ & $0.4085(2)$ & $0.41890(12)$ & $0.70827(7)$ & 0.0495 (4) \\
\hline C31 & $0.2319(2)$ & $0.40506(12)$ & $0.70876(7)$ & $0.0471(4)$ \\
\hline C32 & $0.1502(2)$ & $0.37456(13)$ & $0.65940(8)$ & $0.0544(5)$ \\
\hline H32 & 0.2086 & 0.3632 & 0.6273 & $0.065^{*}$ \\
\hline $\mathrm{C} 33$ & $-0.0126(2)$ & $0.36050(14)$ & $0.65589(9)$ & $0.0603(5)$ \\
\hline H33 & -0.0657 & 0.3407 & 0.6215 & $0.072 *$ \\
\hline $\mathrm{C} 34$ & $-0.0988(2)$ & $0.37524(13)$ & $0.70255(9)$ & $0.0617(5)$ \\
\hline H34 & -0.2113 & 0.3657 & 0.7003 & $0.074 *$ \\
\hline $\mathrm{C} 35$ & $-0.0217(2)$ & $0.40370(14)$ & $0.75227(9)$ & $0.0618(5)$ \\
\hline H35 & -0.0802 & 0.4133 & 0.7846 & $0.074 *$ \\
\hline C36 & $0.1410(2)$ & $0.41815(13)$ & $0.75471(8)$ & $0.0531(5)$ \\
\hline $\mathrm{C} 37$ & $0.4591(3)$ & $0.68400(14)$ & $0.72680(11)$ & $0.0769(7)$ \\
\hline H37A & 0.4104 & 0.7057 & 0.7604 & $0.092 *$ \\
\hline H37B & 0.4061 & 0.7104 & 0.6928 & $0.092 *$ \\
\hline $\mathrm{H} 37 \mathrm{C}$ & 0.5734 & 0.6986 & 0.7293 & $0.092 *$ \\
\hline C38 & $0.5499(3)$ & $0.12902(16)$ & $0.78316(10)$ & $0.0816(7)$ \\
\hline $\mathrm{H} 38 \mathrm{~A}$ & 0.4366 & 0.1457 & 0.7814 & $0.098^{*}$ \\
\hline H38B & 0.5588 & 0.0663 & 0.7862 & $0.098^{*}$ \\
\hline $\mathrm{H} 38 \mathrm{C}$ & 0.5977 & 0.1483 & 0.7488 & $0.098^{*}$ \\
\hline
\end{tabular}

Atomic displacement parameters $\left(\AA^{2}\right)$

\begin{tabular}{lllllll}
\hline & $U^{11}$ & $U^{22}$ & $U^{33}$ & $U^{12}$ & $U^{13}$ & $U^{23}$ \\
\hline F1 & $0.0612(8)$ & $0.0987(9)$ & $0.0760(8)$ & $0.0004(6)$ & $0.0083(6)$ & $0.0368(7)$
\end{tabular}


supporting information

\begin{tabular}{|c|c|c|c|c|c|c|}
\hline F2 & $0.0563(7)$ & $0.1236(11)$ & $0.0527(6)$ & $-0.0066(7)$ & $0.0160(5)$ & $-0.0252(7)$ \\
\hline $\mathrm{O} 1$ & $0.0554(8)$ & $0.0789(9)$ & $0.0521(8)$ & $-0.0110(7)$ & $-0.0022(6)$ & $0.0082(6)$ \\
\hline $\mathrm{O} 2$ & $0.0932(12)$ & $0.0620(9)$ & $0.0689(9)$ & $0.0009(8)$ & $0.0282(8)$ & $-0.0069(7)$ \\
\hline $\mathrm{O} 3$ & $0.0795(10)$ & 0.0635 (9) & $0.0617(8)$ & $-0.0137(7)$ & $0.0117(7)$ & $0.0014(7)$ \\
\hline $\mathrm{O} 4$ & $0.0480(8)$ & $0.0895(10)$ & $0.0488(7)$ & $-0.0059(7)$ & $0.0148(6)$ & $-0.0126(7)$ \\
\hline O5 & $0.0769(10)$ & $0.0601(8)$ & $0.0692(9)$ & $-0.0042(7)$ & $0.0051(8)$ & $-0.0031(7)$ \\
\hline O6 & $0.0879(11)$ & $0.0740(10)$ & $0.0702(10)$ & $0.0165(8)$ & $0.0073(8)$ & $0.0055(8)$ \\
\hline $\mathrm{C} 1$ & $0.0420(10)$ & $0.0636(11)$ & $0.0470(9)$ & $0.0001(8)$ & $0.0084(8)$ & $0.0030(8)$ \\
\hline $\mathrm{C} 2$ & $0.0554(12)$ & $0.0648(12)$ & $0.0553(11)$ & $0.0028(9)$ & $0.0119(9)$ & $0.0020(9)$ \\
\hline $\mathrm{C} 3$ & 0.0667 (14) & $0.0793(15)$ & $0.0562(12)$ & $0.0084(11)$ & $0.0190(10)$ & $-0.0045(10)$ \\
\hline $\mathrm{C} 4$ & $0.0549(13)$ & $0.0885(16)$ & $0.0532(11)$ & $0.0049(11)$ & $0.0184(9)$ & $0.0076(10)$ \\
\hline $\mathrm{C} 5$ & $0.0554(12)$ & $0.0864(15)$ & $0.0531(11)$ & $-0.0083(11)$ & $0.0147(9)$ & $0.0151(11)$ \\
\hline C6 & $0.0633(13)$ & $0.0736(14)$ & $0.0594(12)$ & $-0.0144(11)$ & $0.0085(10)$ & $0.0136(10)$ \\
\hline $\mathrm{C} 7$ & $0.0516(12)$ & $0.0679(13)$ & $0.0527(11)$ & $-0.0075(9)$ & $0.0022(9)$ & $0.0050(9)$ \\
\hline $\mathrm{C} 8$ & 0.0461 (11) & $0.0634(12)$ & $0.0460(10)$ & $-0.0056(8)$ & $0.0061(8)$ & $0.0065(8)$ \\
\hline C9 & $0.0394(10)$ & $0.0665(12)$ & $0.0467(10)$ & $-0.0004(8)$ & $0.0064(8)$ & $0.0067(8)$ \\
\hline $\mathrm{C} 10$ & $0.0438(11)$ & $0.0763(13)$ & $0.0484(10)$ & $0.0002(9)$ & $0.0096(8)$ & $0.0076(9)$ \\
\hline $\mathrm{C} 11$ & $0.0476(11)$ & $0.0539(10)$ & $0.0476(10)$ & $-0.0005(8)$ & $0.0059(8)$ & $0.0008(8)$ \\
\hline $\mathrm{C} 12$ & $0.0458(10)$ & $0.0574(11)$ & $0.0406(9)$ & $-0.0007(8)$ & $0.0087(7)$ & $-0.0033(7)$ \\
\hline $\mathrm{C} 13$ & 0.0508 (11) & $0.0656(12)$ & $0.0486(10)$ & $0.0017(9)$ & $0.0109(8)$ & $0.0035(9)$ \\
\hline C14 & $0.0533(12)$ & $0.0732(13)$ & $0.0632(12)$ & $-0.0033(10)$ & $0.0200(10)$ & $0.0037(10)$ \\
\hline $\mathrm{C} 15$ & $0.0461(12)$ & $0.0832(15)$ & $0.0676(13)$ & $-0.0043(10)$ & $0.0128(10)$ & $-0.0082(11)$ \\
\hline $\mathrm{C} 16$ & $0.0489(12)$ & $0.0879(15)$ & $0.0557(11)$ & $0.0035(10)$ & $0.0005(9)$ & $-0.0022(10)$ \\
\hline $\mathrm{C} 17$ & $0.0533(12)$ & $0.0708(13)$ & $0.0437(10)$ & $-0.0002(9)$ & $0.0064(8)$ & $0.0008(8)$ \\
\hline $\mathrm{C} 18$ & $0.122(2)$ & $0.0680(15)$ & $0.0831(16)$ & $0.0080(14)$ & $0.0304(15)$ & $-0.0129(12)$ \\
\hline C19 & $0.0698(14)$ & $0.0678(13)$ & $0.0658(13)$ & $-0.0097(11)$ & $0.0112(11)$ & $-0.0040(10)$ \\
\hline $\mathrm{C} 20$ & $0.0439(10)$ & $0.0637(12)$ & $0.0491(10)$ & $-0.0043(8)$ & $0.0132(8)$ & $-0.0070(8)$ \\
\hline $\mathrm{C} 21$ & $0.0484(11)$ & $0.0654(12)$ & $0.0536(11)$ & $-0.0058(9)$ & $0.0138(9)$ & $-0.0059(9)$ \\
\hline $\mathrm{C} 22$ & $0.0540(12)$ & $0.0706(13)$ & $0.0652(13)$ & $-0.0119(10)$ & $0.0198(10)$ & $-0.0188(10)$ \\
\hline $\mathrm{C} 23$ & $0.0454(11)$ & 0.0917 (16) & $0.0553(11)$ & $-0.0079(10)$ & $0.0123(9)$ & $-0.0224(11)$ \\
\hline $\mathrm{C} 24$ & $0.0602(14)$ & $0.1036(19)$ & $0.0482(11)$ & 0.0051 (12) & $0.0033(9)$ & $-0.0127(11)$ \\
\hline $\mathrm{C} 25$ & $0.0766(16)$ & $0.0989(19)$ & $0.0481(11)$ & 0.0189 (13) & $0.0052(10)$ & $-0.0022(11)$ \\
\hline $\mathrm{C} 26$ & $0.0578(13)$ & 0.0745 (14) & 0.0507 (11) & $0.0087(10)$ & $0.0117(9)$ & $0.0025(9)$ \\
\hline $\mathrm{C} 27$ & $0.0458(11)$ & $0.0706(12)$ & $0.0469(10)$ & $0.0009(9)$ & $0.0092(8)$ & $-0.0057(9)$ \\
\hline $\mathrm{C} 28$ & $0.0395(10)$ & $0.0709(12)$ & $0.0450(9)$ & $-0.0026(8)$ & $0.0120(8)$ & $-0.0056(9)$ \\
\hline $\mathrm{C} 29$ & $0.0418(11)$ & $0.0839(14)$ & $0.0458(10)$ & $-0.0028(9)$ & $0.0104(8)$ & $-0.0137(9)$ \\
\hline $\mathrm{C} 30$ & 0.0464 (11) & $0.0582(11)$ & $0.0450(10)$ & $-0.0026(8)$ & $0.0103(8)$ & $-0.0005(8)$ \\
\hline $\mathrm{C} 31$ & $0.0419(10)$ & $0.0558(10)$ & $0.0441(9)$ & $-0.0007(8)$ & $0.0068(7)$ & $0.0009(8)$ \\
\hline C32 & 0.0501 (11) & $0.0691(12)$ & $0.0444(10)$ & $-0.0020(9)$ & $0.0063(8)$ & $-0.0001(8)$ \\
\hline $\mathrm{C} 33$ & $0.0456(11)$ & $0.0755(13)$ & $0.0588(12)$ & $-0.0062(9)$ & $-0.0023(9)$ & $-0.0012(10)$ \\
\hline C34 & 0.0403 (11) & $0.0676(13)$ & $0.0775(14)$ & $-0.0030(9)$ & $0.0071(10)$ & $0.0038(10)$ \\
\hline $\mathrm{C} 35$ & $0.0468(12)$ & $0.0749(13)$ & $0.0661(12)$ & $0.0008(9)$ & $0.0205(10)$ & $-0.0022(10)$ \\
\hline $\mathrm{C} 36$ & $0.0469(11)$ & $0.0673(12)$ & $0.0458(10)$ & $0.0000(9)$ & $0.0085(8)$ & $-0.0054(8)$ \\
\hline C37 & $0.0886(17)$ & $0.0541(12)$ & $0.0903(16)$ & $-0.0022(11)$ & $0.0225(13)$ & $-0.0039(11)$ \\
\hline C38 & 0.0952 (19) & $0.0676(14)$ & $0.0817(16)$ & $0.0102(13)$ & $0.0044(14)$ & $-0.0051(12)$ \\
\hline
\end{tabular}


Geometric parameters $\left(\AA,{ }^{\circ}\right)$

\begin{tabular}{|c|c|c|c|}
\hline $\mathrm{F} 1-\mathrm{C} 13$ & $1.349(2)$ & $\mathrm{C} 18-\mathrm{H} 18 \mathrm{~A}$ & 0.9800 \\
\hline $\mathrm{F} 2-\mathrm{C} 36$ & $1.356(2)$ & $\mathrm{C} 18-\mathrm{H} 18 \mathrm{~B}$ & 0.9800 \\
\hline $\mathrm{O} 1-\mathrm{C} 11$ & $1.217(2)$ & $\mathrm{C} 18-\mathrm{H} 18 \mathrm{C}$ & 0.9800 \\
\hline $\mathrm{O} 2-\mathrm{C} 2$ & $1.376(2)$ & C19-H19A & 0.9800 \\
\hline $\mathrm{O} 2-\mathrm{C} 18$ & $1.426(3)$ & $\mathrm{C} 19-\mathrm{H} 19 \mathrm{~B}$ & 0.9800 \\
\hline $\mathrm{O} 3-\mathrm{C} 7$ & $1.357(2)$ & C19-H19C & 0.9800 \\
\hline $\mathrm{O} 3-\mathrm{C} 19$ & $1.427(2)$ & $\mathrm{C} 20-\mathrm{C} 21$ & $1.376(3)$ \\
\hline $\mathrm{O} 4-\mathrm{C} 30$ & $1.216(2)$ & $\mathrm{C} 20-\mathrm{C} 28$ & $1.404(3)$ \\
\hline $\mathrm{O} 5-\mathrm{C} 21$ & $1.372(2)$ & $\mathrm{C} 20-\mathrm{C} 30$ & $1.504(3)$ \\
\hline $\mathrm{O} 5-\mathrm{C} 37$ & $1.437(2)$ & $\mathrm{C} 21-\mathrm{C} 22$ & $1.414(3)$ \\
\hline $\mathrm{O} 6-\mathrm{C} 26$ & $1.376(3)$ & $\mathrm{C} 22-\mathrm{C} 23$ & $1.375(3)$ \\
\hline $\mathrm{O} 6-\mathrm{C} 38$ & $1.421(3)$ & $\mathrm{C} 22-\mathrm{H} 22$ & 0.9500 \\
\hline $\mathrm{C} 1-\mathrm{C} 2$ & $1.378(3)$ & $\mathrm{C} 23-\mathrm{C} 29$ & $1.413(3)$ \\
\hline $\mathrm{C} 1-\mathrm{C} 9$ & $1.419(3)$ & $\mathrm{C} 23-\mathrm{H} 23$ & 0.9500 \\
\hline $\mathrm{C} 1-\mathrm{C} 11$ & $1.504(2)$ & $\mathrm{C} 24-\mathrm{C} 25$ & $1.344(3)$ \\
\hline $\mathrm{C} 2-\mathrm{C} 3$ & $1.407(3)$ & $\mathrm{C} 24-\mathrm{C} 29$ & $1.419(3)$ \\
\hline $\mathrm{C} 3-\mathrm{C} 4$ & $1.358(3)$ & $\mathrm{C} 24-\mathrm{H} 24$ & 0.9500 \\
\hline $\mathrm{C} 3-\mathrm{H} 3$ & 0.9500 & $\mathrm{C} 25-\mathrm{C} 26$ & $1.398(3)$ \\
\hline $\mathrm{C} 4-\mathrm{C} 10$ & $1.410(3)$ & $\mathrm{C} 25-\mathrm{H} 25$ & 0.9500 \\
\hline $\mathrm{C} 4-\mathrm{H} 4$ & 0.9500 & $\mathrm{C} 26-\mathrm{C} 27$ & $1.356(3)$ \\
\hline $\mathrm{C} 5-\mathrm{C} 6$ & $1.348(3)$ & $\mathrm{C} 27-\mathrm{C} 28$ & $1.437(3)$ \\
\hline $\mathrm{C} 5-\mathrm{C} 10$ & $1.419(3)$ & $\mathrm{C} 27-\mathrm{H} 27$ & 0.9500 \\
\hline $\mathrm{C} 5-\mathrm{H} 5$ & 0.9500 & $\mathrm{C} 28-\mathrm{C} 29$ & $1.418(3)$ \\
\hline $\mathrm{C} 6-\mathrm{C} 7$ & $1.413(3)$ & $\mathrm{C} 30-\mathrm{C} 31$ & $1.493(2)$ \\
\hline $\mathrm{C} 6-\mathrm{H} 6$ & 0.9500 & $\mathrm{C} 31-\mathrm{C} 36$ & $1.387(2)$ \\
\hline $\mathrm{C} 7-\mathrm{C} 8$ & $1.377(3)$ & $\mathrm{C} 31-\mathrm{C} 32$ & $1.394(2)$ \\
\hline $\mathrm{C} 8-\mathrm{C} 9$ & $1.417(3)$ & $\mathrm{C} 32-\mathrm{C} 33$ & $1.376(3)$ \\
\hline $\mathrm{C} 8-\mathrm{H} 8$ & 0.9500 & C $32-\mathrm{H} 32$ & 0.9500 \\
\hline $\mathrm{C} 9-\mathrm{C} 10$ & $1.424(2)$ & $\mathrm{C} 33-\mathrm{C} 34$ & $1.381(3)$ \\
\hline $\mathrm{C} 11-\mathrm{C} 12$ & $1.489(3)$ & C $33-\mathrm{H} 33$ & 0.9500 \\
\hline $\mathrm{C} 12-\mathrm{C} 13$ & $1.387(2)$ & $\mathrm{C} 34-\mathrm{C} 35$ & $1.374(3)$ \\
\hline $\mathrm{C} 12-\mathrm{C} 17$ & $1.394(3)$ & C34-H34 & 0.9500 \\
\hline $\mathrm{C} 13-\mathrm{C} 14$ & $1.373(3)$ & $\mathrm{C} 35-\mathrm{C} 36$ & $1.376(3)$ \\
\hline $\mathrm{C} 14-\mathrm{C} 15$ & $1.369(3)$ & C35-H35 & 0.9500 \\
\hline C14-H14 & 0.9500 & C37-H37A & 0.9800 \\
\hline $\mathrm{C} 15-\mathrm{C} 16$ & $1.384(3)$ & C37-H37B & 0.9800 \\
\hline C15-H15 & 0.9500 & C37-H37C & 0.9800 \\
\hline $\mathrm{C} 16-\mathrm{C} 17$ & $1.383(3)$ & C $38-\mathrm{H} 38 \mathrm{~A}$ & 0.9800 \\
\hline $\mathrm{C} 16-\mathrm{H} 16$ & 0.9500 & C38-H38B & 0.9800 \\
\hline $\mathrm{C} 17-\mathrm{H} 17$ & 0.9500 & $\mathrm{C} 38-\mathrm{H} 38 \mathrm{C}$ & 0.9800 \\
\hline $\mathrm{C} 2-\mathrm{O} 2-\mathrm{C} 18$ & $118.02(17)$ & $\mathrm{H} 19 \mathrm{~A}-\mathrm{C} 19-\mathrm{H} 19 \mathrm{C}$ & 109.5 \\
\hline $\mathrm{C} 7-\mathrm{O} 3-\mathrm{C} 19$ & $116.96(16)$ & $\mathrm{H} 19 \mathrm{~B}-\mathrm{C} 19-\mathrm{H} 19 \mathrm{C}$ & 109.5 \\
\hline $\mathrm{C} 21-\mathrm{O} 5-\mathrm{C} 37$ & $117.89(17)$ & $\mathrm{C} 21-\mathrm{C} 20-\mathrm{C} 28$ & $120.44(17)$ \\
\hline $\mathrm{C} 26-\mathrm{O} 6-\mathrm{C} 38$ & $117.49(17)$ & $\mathrm{C} 21-\mathrm{C} 20-\mathrm{C} 30$ & $120.50(18)$ \\
\hline $\mathrm{C} 2-\mathrm{C} 1-\mathrm{C} 9$ & $120.35(17)$ & $\mathrm{C} 28-\mathrm{C} 20-\mathrm{C} 30$ & $119.05(17)$ \\
\hline
\end{tabular}




\begin{tabular}{|c|c|c|c|}
\hline $\mathrm{C} 2-\mathrm{C} 1-\mathrm{C} 11$ & $118.30(17)$ & $\mathrm{O} 5-\mathrm{C} 21-\mathrm{C} 20$ & $115.47(17)$ \\
\hline $\mathrm{C} 9-\mathrm{C} 1-\mathrm{C} 11$ & $121.24(16)$ & $\mathrm{O} 5-\mathrm{C} 21-\mathrm{C} 22$ & $123.80(19)$ \\
\hline $\mathrm{O} 2-\mathrm{C} 2-\mathrm{C} 1$ & $115.46(17)$ & $\mathrm{C} 20-\mathrm{C} 21-\mathrm{C} 22$ & $120.7(2)$ \\
\hline $\mathrm{O} 2-\mathrm{C} 2-\mathrm{C} 3$ & $123.34(19)$ & $\mathrm{C} 23-\mathrm{C} 22-\mathrm{C} 21$ & $119.4(2)$ \\
\hline $\mathrm{C} 1-\mathrm{C} 2-\mathrm{C} 3$ & $121.18(19)$ & $\mathrm{C} 23-\mathrm{C} 22-\mathrm{H} 22$ & 120.3 \\
\hline $\mathrm{C} 4-\mathrm{C} 3-\mathrm{C} 2$ & $118.9(2)$ & $\mathrm{C} 21-\mathrm{C} 22-\mathrm{H} 22$ & 120.3 \\
\hline $\mathrm{C} 4-\mathrm{C} 3-\mathrm{H} 3$ & 120.5 & $\mathrm{C} 22-\mathrm{C} 23-\mathrm{C} 29$ & $121.13(19)$ \\
\hline $\mathrm{C} 2-\mathrm{C} 3-\mathrm{H} 3$ & 120.5 & $\mathrm{C} 22-\mathrm{C} 23-\mathrm{H} 23$ & 119.4 \\
\hline $\mathrm{C} 3-\mathrm{C} 4-\mathrm{C} 10$ & $122.35(18)$ & $\mathrm{C} 29-\mathrm{C} 23-\mathrm{H} 23$ & 119.4 \\
\hline $\mathrm{C} 3-\mathrm{C} 4-\mathrm{H} 4$ & 118.8 & $\mathrm{C} 25-\mathrm{C} 24-\mathrm{C} 29$ & $121.7(2)$ \\
\hline $\mathrm{C} 10-\mathrm{C} 4-\mathrm{H} 4$ & 118.8 & $\mathrm{C} 25-\mathrm{C} 24-\mathrm{H} 24$ & 119.1 \\
\hline $\mathrm{C} 6-\mathrm{C} 5-\mathrm{C} 10$ & $121.17(18)$ & $\mathrm{C} 29-\mathrm{C} 24-\mathrm{H} 24$ & 119.1 \\
\hline $\mathrm{C} 6-\mathrm{C} 5-\mathrm{H} 5$ & 119.4 & $\mathrm{C} 24-\mathrm{C} 25-\mathrm{C} 26$ & $120.3(2)$ \\
\hline $\mathrm{C} 10-\mathrm{C} 5-\mathrm{H} 5$ & 119.4 & $\mathrm{C} 24-\mathrm{C} 25-\mathrm{H} 25$ & 119.9 \\
\hline $\mathrm{C} 5-\mathrm{C} 6-\mathrm{C} 7$ & $120.4(2)$ & $\mathrm{C} 26-\mathrm{C} 25-\mathrm{H} 25$ & 119.9 \\
\hline $\mathrm{C} 5-\mathrm{C} 6-\mathrm{H} 6$ & 119.8 & $\mathrm{C} 27-\mathrm{C} 26-\mathrm{O} 6$ & $124.01(19)$ \\
\hline $\mathrm{C} 7-\mathrm{C} 6-\mathrm{H} 6$ & 119.8 & $\mathrm{C} 27-\mathrm{C} 26-\mathrm{C} 25$ & $121.5(2)$ \\
\hline $\mathrm{O} 3-\mathrm{C} 7-\mathrm{C} 8$ & 125.14 (18) & $\mathrm{O} 6-\mathrm{C} 26-\mathrm{C} 25$ & $114.49(19)$ \\
\hline $\mathrm{O} 3-\mathrm{C} 7-\mathrm{C} 6$ & $114.37(18)$ & $\mathrm{C} 26-\mathrm{C} 27-\mathrm{C} 28$ & $119.36(18)$ \\
\hline $\mathrm{C} 8-\mathrm{C} 7-\mathrm{C} 6$ & 120.49 (19) & $\mathrm{C} 26-\mathrm{C} 27-\mathrm{H} 27$ & 120.3 \\
\hline $\mathrm{C} 7-\mathrm{C} 8-\mathrm{C} 9$ & $120.04(17)$ & $\mathrm{C} 28-\mathrm{C} 27-\mathrm{H} 27$ & 120.3 \\
\hline $\mathrm{C} 7-\mathrm{C} 8-\mathrm{H} 8$ & 120.0 & $\mathrm{C} 20-\mathrm{C} 28-\mathrm{C} 29$ & $119.44(19)$ \\
\hline $\mathrm{C} 9-\mathrm{C} 8-\mathrm{H} 8$ & 120.0 & $\mathrm{C} 20-\mathrm{C} 28-\mathrm{C} 27$ & $121.40(17)$ \\
\hline $\mathrm{C} 8-\mathrm{C} 9-\mathrm{C} 1$ & $122.51(16)$ & $\mathrm{C} 29-\mathrm{C} 28-\mathrm{C} 27$ & $119.17(18)$ \\
\hline $\mathrm{C} 8-\mathrm{C} 9-\mathrm{C} 10$ & $119.07(17)$ & $\mathrm{C} 23-\mathrm{C} 29-\mathrm{C} 28$ & $118.88(19)$ \\
\hline $\mathrm{C} 1-\mathrm{C} 9-\mathrm{C} 10$ & $118.41(18)$ & $\mathrm{C} 23-\mathrm{C} 29-\mathrm{C} 24$ & $123.12(19)$ \\
\hline $\mathrm{C} 4-\mathrm{C} 10-\mathrm{C} 5$ & $122.54(18)$ & $\mathrm{C} 28-\mathrm{C} 29-\mathrm{C} 24$ & $118.0(2)$ \\
\hline $\mathrm{C} 4-\mathrm{C} 10-\mathrm{C} 9$ & $118.76(18)$ & $\mathrm{O} 4-\mathrm{C} 30-\mathrm{C} 31$ & $119.49(16)$ \\
\hline $\mathrm{C} 5-\mathrm{C} 10-\mathrm{C} 9$ & $118.70(19)$ & $\mathrm{O} 4-\mathrm{C} 30-\mathrm{C} 20$ & $119.50(16)$ \\
\hline $\mathrm{O} 1-\mathrm{C} 11-\mathrm{C} 12$ & $121.72(16)$ & $\mathrm{C} 31-\mathrm{C} 30-\mathrm{C} 20$ & $120.92(14)$ \\
\hline $\mathrm{O} 1-\mathrm{C} 11-\mathrm{C} 1$ & $120.24(16)$ & $\mathrm{C} 36-\mathrm{C} 31-\mathrm{C} 32$ & $116.43(16)$ \\
\hline $\mathrm{C} 12-\mathrm{C} 11-\mathrm{C} 1$ & $118.04(15)$ & $\mathrm{C} 36-\mathrm{C} 31-\mathrm{C} 30$ & $125.70(16)$ \\
\hline $\mathrm{C} 13-\mathrm{C} 12-\mathrm{C} 17$ & $116.63(17)$ & $\mathrm{C} 32-\mathrm{C} 31-\mathrm{C} 30$ & $117.85(15)$ \\
\hline $\mathrm{C} 13-\mathrm{C} 12-\mathrm{C} 11$ & $122.78(16)$ & $\mathrm{C} 33-\mathrm{C} 32-\mathrm{C} 31$ & $121.83(17)$ \\
\hline $\mathrm{C} 17-\mathrm{C} 12-\mathrm{C} 11$ & $120.59(16)$ & $\mathrm{C} 33-\mathrm{C} 32-\mathrm{H} 32$ & 119.1 \\
\hline $\mathrm{F} 1-\mathrm{C} 13-\mathrm{C} 14$ & $117.43(17)$ & $\mathrm{C} 31-\mathrm{C} 32-\mathrm{H} 32$ & 119.1 \\
\hline $\mathrm{F} 1-\mathrm{C} 13-\mathrm{C} 12$ & $119.34(17)$ & $\mathrm{C} 32-\mathrm{C} 33-\mathrm{C} 34$ & $119.77(19)$ \\
\hline $\mathrm{C} 14-\mathrm{C} 13-\mathrm{C} 12$ & $123.14(18)$ & $\mathrm{C} 32-\mathrm{C} 33-\mathrm{H} 33$ & 120.1 \\
\hline $\mathrm{C} 15-\mathrm{C} 14-\mathrm{C} 13$ & $118.77(19)$ & $\mathrm{C} 34-\mathrm{C} 33-\mathrm{H} 33$ & 120.1 \\
\hline $\mathrm{C} 15-\mathrm{C} 14-\mathrm{H} 14$ & 120.6 & $\mathrm{C} 35-\mathrm{C} 34-\mathrm{C} 33$ & $120.02(19)$ \\
\hline $\mathrm{C} 13-\mathrm{C} 14-\mathrm{H} 14$ & 120.6 & $\mathrm{C} 35-\mathrm{C} 34-\mathrm{H} 34$ & 120.0 \\
\hline $\mathrm{C} 14-\mathrm{C} 15-\mathrm{C} 16$ & $120.52(19)$ & $\mathrm{C} 33-\mathrm{C} 34-\mathrm{H} 34$ & 120.0 \\
\hline $\mathrm{C} 14-\mathrm{C} 15-\mathrm{H} 15$ & 119.7 & $\mathrm{C} 34-\mathrm{C} 35-\mathrm{C} 36$ & $119.28(18)$ \\
\hline $\mathrm{C} 16-\mathrm{C} 15-\mathrm{H} 15$ & 119.7 & $\mathrm{C} 34-\mathrm{C} 35-\mathrm{H} 35$ & 120.4 \\
\hline $\mathrm{C} 17-\mathrm{C} 16-\mathrm{C} 15$ & $119.7(2)$ & $\mathrm{C} 36-\mathrm{C} 35-\mathrm{H} 35$ & 120.4 \\
\hline $\mathrm{C} 17-\mathrm{C} 16-\mathrm{H} 16$ & 120.1 & $\mathrm{~F} 2-\mathrm{C} 36-\mathrm{C} 35$ & $117.15(16)$ \\
\hline $\mathrm{C} 15-\mathrm{C} 16-\mathrm{H} 16$ & 120.1 & $\mathrm{~F} 2-\mathrm{C} 36-\mathrm{C} 31$ & $120.21(16)$ \\
\hline $\mathrm{C} 16-\mathrm{C} 17-\mathrm{C} 12$ & $121.20(18)$ & $\mathrm{C} 35-\mathrm{C} 36-\mathrm{C} 31$ & $122.65(18)$ \\
\hline
\end{tabular}




\begin{tabular}{|c|c|c|c|}
\hline $\mathrm{C} 16-\mathrm{C} 17-\mathrm{H} 17$ & 119.4 & $\mathrm{O} 5-\mathrm{C} 37-\mathrm{H} 37 \mathrm{~A}$ & 109.5 \\
\hline $\mathrm{C} 12-\mathrm{C} 17-\mathrm{H} 17$ & 119.4 & $\mathrm{O} 5-\mathrm{C} 37-\mathrm{H} 37 \mathrm{~B}$ & 109.5 \\
\hline $\mathrm{O} 2-\mathrm{C} 18-\mathrm{H} 18 \mathrm{~A}$ & 109.5 & $\mathrm{H} 37 \mathrm{~A}-\mathrm{C} 37-\mathrm{H} 37 \mathrm{~B}$ & 109.5 \\
\hline $\mathrm{O} 2-\mathrm{C} 18-\mathrm{H} 18 \mathrm{~B}$ & 109.5 & $\mathrm{O} 5-\mathrm{C} 37-\mathrm{H} 37 \mathrm{C}$ & 109.5 \\
\hline $\mathrm{H} 18 \mathrm{~A}-\mathrm{C} 18-\mathrm{H} 18 \mathrm{~B}$ & 109.5 & $\mathrm{H} 37 \mathrm{~A}-\mathrm{C} 37-\mathrm{H} 37 \mathrm{C}$ & 109.5 \\
\hline $\mathrm{O} 2-\mathrm{C} 18-\mathrm{H} 18 \mathrm{C}$ & 109.5 & $\mathrm{H} 37 \mathrm{~B}-\mathrm{C} 37-\mathrm{H} 37 \mathrm{C}$ & 109.5 \\
\hline $\mathrm{H} 18 \mathrm{~A}-\mathrm{C} 18-\mathrm{H} 18 \mathrm{C}$ & 109.5 & $\mathrm{O} 6-\mathrm{C} 38-\mathrm{H} 38 \mathrm{~A}$ & 109.5 \\
\hline $\mathrm{H} 18 \mathrm{~B}-\mathrm{C} 18-\mathrm{H} 18 \mathrm{C}$ & 109.5 & $\mathrm{O} 6-\mathrm{C} 38-\mathrm{H} 38 \mathrm{~B}$ & 109.5 \\
\hline $\mathrm{O} 3-\mathrm{C} 19-\mathrm{H} 19 \mathrm{~A}$ & 109.5 & $\mathrm{H} 38 \mathrm{~A}-\mathrm{C} 38-\mathrm{H} 38 \mathrm{~B}$ & 109.5 \\
\hline $\mathrm{O} 3-\mathrm{C} 19-\mathrm{H} 19 \mathrm{~B}$ & 109.5 & $\mathrm{O} 6-\mathrm{C} 38-\mathrm{H} 38 \mathrm{C}$ & 109.5 \\
\hline $\mathrm{H} 19 \mathrm{~A}-\mathrm{C} 19-\mathrm{H} 19 \mathrm{~B}$ & 109.5 & $\mathrm{H} 38 \mathrm{~A}-\mathrm{C} 38-\mathrm{H} 38 \mathrm{C}$ & 109.5 \\
\hline $\mathrm{O} 3-\mathrm{C} 19-\mathrm{H} 19 \mathrm{C}$ & 109.5 & $\mathrm{H} 38 \mathrm{~B}-\mathrm{C} 38-\mathrm{H} 38 \mathrm{C}$ & 109.5 \\
\hline $\mathrm{C} 18-\mathrm{O} 2-\mathrm{C} 2-\mathrm{C} 1$ & $-174.3(2)$ & $\mathrm{C} 37-\mathrm{O} 5-\mathrm{C} 21-\mathrm{C} 20$ & $-177.68(17)$ \\
\hline $\mathrm{C} 18-\mathrm{O} 2-\mathrm{C} 2-\mathrm{C} 3$ & $3.9(3)$ & $\mathrm{C} 37-\mathrm{O} 5-\mathrm{C} 21-\mathrm{C} 22$ & $3.2(3)$ \\
\hline $\mathrm{C} 9-\mathrm{C} 1-\mathrm{C} 2-\mathrm{O} 2$ & $179.53(17)$ & $\mathrm{C} 28-\mathrm{C} 20-\mathrm{C} 21-\mathrm{O} 5$ & $-178.40(16)$ \\
\hline $\mathrm{C} 11-\mathrm{C} 1-\mathrm{C} 2-\mathrm{O} 2$ & $3.4(3)$ & $\mathrm{C} 30-\mathrm{C} 20-\mathrm{C} 21-\mathrm{O} 5$ & $2.6(2)$ \\
\hline $\mathrm{C} 9-\mathrm{C} 1-\mathrm{C} 2-\mathrm{C} 3$ & $1.2(3)$ & $\mathrm{C} 28-\mathrm{C} 20-\mathrm{C} 21-\mathrm{C} 22$ & $0.7(3)$ \\
\hline $\mathrm{C} 11-\mathrm{C} 1-\mathrm{C} 2-\mathrm{C} 3$ & $-174.89(18)$ & $\mathrm{C} 30-\mathrm{C} 20-\mathrm{C} 21-\mathrm{C} 22$ & $-178.31(16)$ \\
\hline $\mathrm{O} 2-\mathrm{C} 2-\mathrm{C} 3-\mathrm{C} 4$ & $-178.3(2)$ & $\mathrm{O} 5-\mathrm{C} 21-\mathrm{C} 22-\mathrm{C} 23$ & $177.09(17)$ \\
\hline $\mathrm{C} 1-\mathrm{C} 2-\mathrm{C} 3-\mathrm{C} 4$ & $-0.2(3)$ & $\mathrm{C} 20-\mathrm{C} 21-\mathrm{C} 22-\mathrm{C} 23$ & $-2.0(3)$ \\
\hline $\mathrm{C} 2-\mathrm{C} 3-\mathrm{C} 4-\mathrm{C} 10$ & $-0.4(3)$ & $\mathrm{C} 21-\mathrm{C} 22-\mathrm{C} 23-\mathrm{C} 29$ & $1.3(3)$ \\
\hline $\mathrm{C} 10-\mathrm{C} 5-\mathrm{C} 6-\mathrm{C} 7$ & $-0.1(3)$ & $\mathrm{C} 29-\mathrm{C} 24-\mathrm{C} 25-\mathrm{C} 26$ & $-0.6(3)$ \\
\hline $\mathrm{C} 19-\mathrm{O} 3-\mathrm{C} 7-\mathrm{C} 8$ & $-4.5(3)$ & $\mathrm{C} 38-\mathrm{O} 6-\mathrm{C} 26-\mathrm{C} 27$ & $1.9(3)$ \\
\hline $\mathrm{C} 19-\mathrm{O} 3-\mathrm{C} 7-\mathrm{C} 6$ & $175.66(18)$ & $\mathrm{C} 38-\mathrm{O} 6-\mathrm{C} 26-\mathrm{C} 25$ & $-178.0(2)$ \\
\hline $\mathrm{C} 5-\mathrm{C} 6-\mathrm{C} 7-\mathrm{O} 3$ & $-177.94(19)$ & $\mathrm{C} 24-\mathrm{C} 25-\mathrm{C} 26-\mathrm{C} 27$ & $0.0(3)$ \\
\hline $\mathrm{C} 5-\mathrm{C} 6-\mathrm{C} 7-\mathrm{C} 8$ & $2.2(3)$ & $\mathrm{C} 24-\mathrm{C} 25-\mathrm{C} 26-\mathrm{O} 6$ & $180.0(2)$ \\
\hline $\mathrm{O} 3-\mathrm{C} 7-\mathrm{C} 8-\mathrm{C} 9$ & $178.56(18)$ & $\mathrm{O} 6-\mathrm{C} 26-\mathrm{C} 27-\mathrm{C} 28$ & $-179.71(17)$ \\
\hline $\mathrm{C} 6-\mathrm{C} 7-\mathrm{C} 8-\mathrm{C} 9$ & $-1.6(3)$ & $\mathrm{C} 25-\mathrm{C} 26-\mathrm{C} 27-\mathrm{C} 28$ & $0.2(3)$ \\
\hline $\mathrm{C} 7-\mathrm{C} 8-\mathrm{C} 9-\mathrm{C} 1$ & $-179.65(18)$ & $\mathrm{C} 21-\mathrm{C} 20-\mathrm{C} 28-\mathrm{C} 29$ & $1.2(3)$ \\
\hline $\mathrm{C} 7-\mathrm{C} 8-\mathrm{C} 9-\mathrm{C} 10$ & $-1.0(3)$ & $\mathrm{C} 30-\mathrm{C} 20-\mathrm{C} 28-\mathrm{C} 29$ & $-179.77(15)$ \\
\hline $\mathrm{C} 2-\mathrm{C} 1-\mathrm{C} 9-\mathrm{C} 8$ & $176.93(18)$ & $\mathrm{C} 21-\mathrm{C} 20-\mathrm{C} 28-\mathrm{C} 27$ & $-178.46(16)$ \\
\hline $\mathrm{C} 11-\mathrm{C} 1-\mathrm{C} 9-\mathrm{C} 8$ & $-7.1(3)$ & $\mathrm{C} 30-\mathrm{C} 20-\mathrm{C} 28-\mathrm{C} 27$ & $0.6(2)$ \\
\hline $\mathrm{C} 2-\mathrm{C} 1-\mathrm{C} 9-\mathrm{C} 10$ & $-1.7(3)$ & $\mathrm{C} 26-\mathrm{C} 27-\mathrm{C} 28-\mathrm{C} 20$ & $179.75(17)$ \\
\hline $\mathrm{C} 11-\mathrm{C} 1-\mathrm{C} 9-\mathrm{C} 10$ & $174.33(17)$ & $\mathrm{C} 26-\mathrm{C} 27-\mathrm{C} 28-\mathrm{C} 29$ & $0.1(3)$ \\
\hline $\mathrm{C} 3-\mathrm{C} 4-\mathrm{C} 10-\mathrm{C} 5$ & $179.3(2)$ & $\mathrm{C} 22-\mathrm{C} 23-\mathrm{C} 29-\mathrm{C} 28$ & $0.6(3)$ \\
\hline $\mathrm{C} 3-\mathrm{C} 4-\mathrm{C} 10-\mathrm{C} 9$ & $-0.1(3)$ & $\mathrm{C} 22-\mathrm{C} 23-\mathrm{C} 29-\mathrm{C} 24$ & $178.98(18)$ \\
\hline $\mathrm{C} 6-\mathrm{C} 5-\mathrm{C} 10-\mathrm{C} 4$ & $178.1(2)$ & $\mathrm{C} 20-\mathrm{C} 28-\mathrm{C} 29-\mathrm{C} 23$ & $-1.8(3)$ \\
\hline $\mathrm{C} 6-\mathrm{C} 5-\mathrm{C} 10-\mathrm{C} 9$ & $-2.5(3)$ & $\mathrm{C} 27-\mathrm{C} 28-\mathrm{C} 29-\mathrm{C} 23$ & $177.82(16)$ \\
\hline $\mathrm{C} 8-\mathrm{C} 9-\mathrm{C} 10-\mathrm{C} 4$ & $-177.56(18)$ & $\mathrm{C} 20-\mathrm{C} 28-\mathrm{C} 29-\mathrm{C} 24$ & $179.71(17)$ \\
\hline $\mathrm{C} 1-\mathrm{C} 9-\mathrm{C} 10-\mathrm{C} 4$ & $1.1(3)$ & $\mathrm{C} 27-\mathrm{C} 28-\mathrm{C} 29-\mathrm{C} 24$ & $-0.7(3)$ \\
\hline $\mathrm{C} 8-\mathrm{C} 9-\mathrm{C} 10-\mathrm{C} 5$ & $3.1(3)$ & $\mathrm{C} 25-\mathrm{C} 24-\mathrm{C} 29-\mathrm{C} 23$ & $-177.5(2)$ \\
\hline $\mathrm{C} 1-\mathrm{C} 9-\mathrm{C} 10-\mathrm{C} 5$ & $-178.28(18)$ & $\mathrm{C} 25-\mathrm{C} 24-\mathrm{C} 29-\mathrm{C} 28$ & $0.9(3)$ \\
\hline $\mathrm{C} 2-\mathrm{C} 1-\mathrm{C} 11-\mathrm{O} 1$ & $102.8(2)$ & $\mathrm{C} 21-\mathrm{C} 20-\mathrm{C} 30-\mathrm{O} 4$ & $95.2(2)$ \\
\hline $\mathrm{C} 9-\mathrm{C} 1-\mathrm{C} 11-\mathrm{O} 1$ & $-73.3(2)$ & $\mathrm{C} 28-\mathrm{C} 20-\mathrm{C} 30-\mathrm{O} 4$ & $-83.9(2)$ \\
\hline $\mathrm{C} 2-\mathrm{C} 1-\mathrm{C} 11-\mathrm{C} 12$ & $-76.9(2)$ & $\mathrm{C} 21-\mathrm{C} 20-\mathrm{C} 30-\mathrm{C} 31$ & $-88.1(2)$ \\
\hline $\mathrm{C} 9-\mathrm{C} 1-\mathrm{C} 11-\mathrm{C} 12$ & $107.1(2)$ & $\mathrm{C} 28-\mathrm{C} 20-\mathrm{C} 30-\mathrm{C} 31$ & $92.8(2)$ \\
\hline $\mathrm{O} 1-\mathrm{C} 11-\mathrm{C} 12-\mathrm{C} 13$ & $-29.1(3)$ & $\mathrm{O} 4-\mathrm{C} 30-\mathrm{C} 31-\mathrm{C} 36$ & $172.05(19)$ \\
\hline
\end{tabular}


$\mathrm{C} 1-\mathrm{C} 11-\mathrm{C} 12-\mathrm{C} 13$

$\mathrm{O} 1-\mathrm{C} 11-\mathrm{C} 12-\mathrm{C} 17$

$\mathrm{C} 1-\mathrm{C} 11-\mathrm{C} 12-\mathrm{C} 17$

$\mathrm{C} 17-\mathrm{C} 12-\mathrm{C} 13-\mathrm{F} 1$

$\mathrm{C} 11-\mathrm{C} 12-\mathrm{C} 13-\mathrm{F} 1$

$\mathrm{C} 17-\mathrm{C} 12-\mathrm{C} 13-\mathrm{C} 14$

$\mathrm{C} 11-\mathrm{C} 12-\mathrm{C} 13-\mathrm{C} 14$

$\mathrm{F} 1-\mathrm{C} 13-\mathrm{C} 14-\mathrm{C} 15$

$\mathrm{C} 12-\mathrm{C} 13-\mathrm{C} 14-\mathrm{C} 15$

$\mathrm{C} 13-\mathrm{C} 14-\mathrm{C} 15-\mathrm{C} 16$

$\mathrm{C} 14-\mathrm{C} 15-\mathrm{C} 16-\mathrm{C} 17$

$\mathrm{C} 15-\mathrm{C} 16-\mathrm{C} 17-\mathrm{C} 12$

$\mathrm{C} 13-\mathrm{C} 12-\mathrm{C} 17-\mathrm{C} 16$

$\mathrm{C} 11-\mathrm{C} 12-\mathrm{C} 17-\mathrm{C} 16$
$150.62(18)$

$150.16(19)$

$-30.2(3)$

177.37 (17)

$-3.4(3)$

$0.8(3)$

$-179.98(18)$

-178.09 (18)

$-1.4(3)$

$0.9(3)$

$0.2(3)$

-0.9 (3)

$0.4(3)$

$-178.86(18)$

$$
\begin{aligned}
& \mathrm{C} 20-\mathrm{C} 30-\mathrm{C} 31-\mathrm{C} 36 \\
& \mathrm{O} 4-\mathrm{C} 30-\mathrm{C} 31-\mathrm{C} 32 \\
& \mathrm{C} 20-\mathrm{C} 30-\mathrm{C} 31-\mathrm{C} 32 \\
& \mathrm{C} 36-\mathrm{C} 31-\mathrm{C} 32-\mathrm{C} 33 \\
& \mathrm{C} 30-\mathrm{C} 31-\mathrm{C} 32-\mathrm{C} 33 \\
& \mathrm{C} 31-\mathrm{C} 32-\mathrm{C} 33-\mathrm{C} 34 \\
& \mathrm{C} 32-\mathrm{C} 33-\mathrm{C} 34-\mathrm{C} 35 \\
& \mathrm{C} 33-\mathrm{C} 34-\mathrm{C} 35-\mathrm{C} 36 \\
& \mathrm{C} 34-\mathrm{C} 35-\mathrm{C} 36-\mathrm{F} 2 \\
& \mathrm{C} 34-\mathrm{C} 35-\mathrm{C} 36-\mathrm{C} 31 \\
& \mathrm{C} 32-\mathrm{C} 31-\mathrm{C} 36-\mathrm{F} 2 \\
& \mathrm{C} 30-\mathrm{C} 31-\mathrm{C} 36-\mathrm{F} 2 \\
& \mathrm{C} 32-\mathrm{C} 31-\mathrm{C} 36-\mathrm{C} 35 \\
& \mathrm{C} 30-\mathrm{C} 31-\mathrm{C} 36-\mathrm{C} 35
\end{aligned}
$$

